\title{
Object Detection in Image with Complex Background
}

\author{
Dong Li, Yali Li, Fei He, Shengjin Wang ${ }^{1}$ \\ State Key Laboratory of Intelligent Technology and Systems \\ Tsinghua National Laboratory for Information Science and Technology \\ Department of Electronic Engineering, Tsinghua University, Beijing China
}

\begin{abstract}
Object detection is the key technology in computer vision, with broad application prospects. Object detection has great research value and practical significance as a hot spot of video surveillance in recent years. This paper proposes an algorithm for ship detection in image with complex harbor background. We test the performance of several texture descriptors, and a region growing method based on contrast texture feature is proposed to implement sea-land separation. Then, we apply a method combined with adaptive threshold segmentation and shape analysis for offshore ship detection. Furthermore, the salient boundary template matching in the sea-land border area is used for docked ship detection. The experimental results show that our algorithm is able to implement ship object detection in complex image with good robustness and real-time performance.
\end{abstract}

Keywords: Object detection • Ship detection • Video surveillance • Complex Background

\section{Introduction}

Object detection is the key technology in computer vision, with broad application prospects. Object detection has great research value and practical significance as a hot spot of video surveillance in recent years. The ship is a kind of important marine transharboration, and ship detection is widely used in many fields. For example, government and port enterprises focus on the security management and risk control of dangerous ships in port operations. In addition, ship detection plays an important role in counting the number of ships and port throughput evaluation. In recent years, with the development of intelligent video surveillance technology,

\footnotetext{
${ }^{1}$ Shengjin Wang $(\square)$

State Key Laboratory of Intelligent Technology and Systems

Tsinghua National Laboratory for Information Science and Technology

Department of Electronic Engineering, Tsinghua University, Beijing 100084,

e-mail:wsj@ocrserv.ee.tsinghua.edu.cn
} China 
ship detection has aroused more and more concern [2]. Study of elimination process of false alarms is both the emphasis and difficulty in ship detection in the top view. Zhu et al. [1] proposed a method of ship detection from spaceborne optical image based on shape and texture features, using simple shape analysis and a novel semisupervised hierarchical classification approach. In [6] the sparseness and appearance of ships on the sea was considered for reducing false alarms. The fine texture and shape features are beneficial to the performance of ship detection for high-resolution remote sensing images [4]. Background clutter has a great influence on the performance of ship detection algorithm in the front view. An effective method for ship detection in sea background is ship segmentation in the regions of interest based on sea-sky line detection [3]. Liu et al. [5] proposed an interactive infrared ship segmentation method based on graph cut and fuzzy connectedness, which is able to handle slightly more complex situations as a result of interaction. Our work focuses on ship object detection in image with complex harbor background. A plurality of interfering factors exist in complex harbor image, such as wharfs, clouds, waves, islands. If we directly adopt the sliding window approach to search and match ship targets in the whole harbor image, it will produce too many false alarms and fail to perform ship detection.

This paper proposes an algorithm for ship detection in image with complex harbor background. We make good use of prior knowledge and image information, including gray, texture and shape features, to detect ship object. The experimental results show that our algorithm is able to implement ship object detection in complex image with good robustness and real-time performance.

This paper is organized as follows. The second part gives a detailed introduction of our ship detection algorithm in image with complex harbor background. The experimental results are proposed and analyzed in the third part. The fourth part summarizes this paper.

\section{Proposed Methodology}

In this section, we will introduce our ship detection algorithm in detail. Our algorithm consists of three main parts: sea-land separation, offshore ship detection and docked ship detection. The flow chart of our algorithm is shown as Fig. 1.

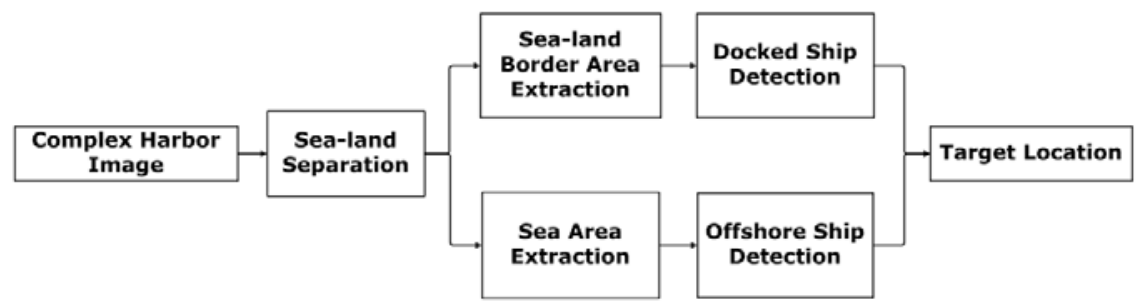

Fig. 1 The flow chart of our algorithm 


\subsection{Sea-land Separation}

Texture is an attribute of image based on regions instead of pixels. We implement sea-land separation based on the texture difference between the sea and land for purpose of eliminating false alarms on the land.

Several common texture descriptors based on grayscale histogram are defined as follows:

Contrast:

$$
f_{1}=\sqrt{\sum_{i=0}^{L-1}\left(z_{i}-m\right)^{2} p\left(z_{i}\right)}
$$

Smoothness:

$$
f_{2}=1-\frac{1}{1+f_{1}^{2}}
$$

Third moment:

$$
f_{3}=\sum_{i=0}^{L-1}\left(z_{i}-m\right)^{3} p\left(z_{i}\right)
$$

Uniformity:

$$
f_{4}=\sum_{i=0}^{L-1} p^{2}\left(z_{i}\right)
$$

Entropy:

$$
f_{5}=-\sum_{i=0}^{L-1} p\left(z_{i}\right) \operatorname{lo}_{2} g p\left(z_{i}\right)
$$

Several common texture descriptors based on gray level co-occurrence matrix are defined as follows:

Contrast:

$$
f_{6}=\sum_{i, j}|i-j|^{2} g(i, j)
$$

Energy:

$$
f_{7}=\sum_{i, j} g^{2}(i, j)
$$

Homogeneity:

$$
f_{8}=\sum_{i, j} \frac{g(i, j)}{1+|i-j|}
$$

Combining texture features, We apply a region growing method to segment the sea and land. The details of our proposed method is described below. Firstly, the image is divided into $\mathrm{N}^{*} \mathrm{M}$ spatial cells and each of them consists of $\mathrm{k}^{*} \mathrm{k}$ pixels. An initial cell is selected as the seed according to the prior knowledge and added to a linear queue. During each pass in the loop, we take an cell from the queue successively and compute the texture feature similarity between its adjacent cells 
which haven't been computed and the seed. When the similarity exceeds a certain threshold, it denotes that the current cell may belong to the sea area and will be added to the queue. The texture feature of the seed will be constantly updated with the mean value in the process. The algorithm will be terminated until the cells which meet the growth conditions can not be found. Our algorithm diagram is shown in Fig. 2.

(a)

Image Region

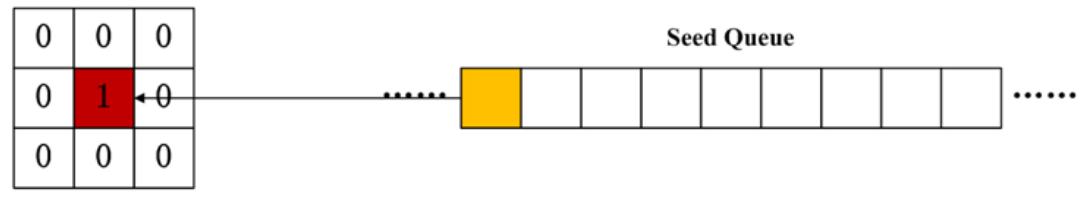

(b)

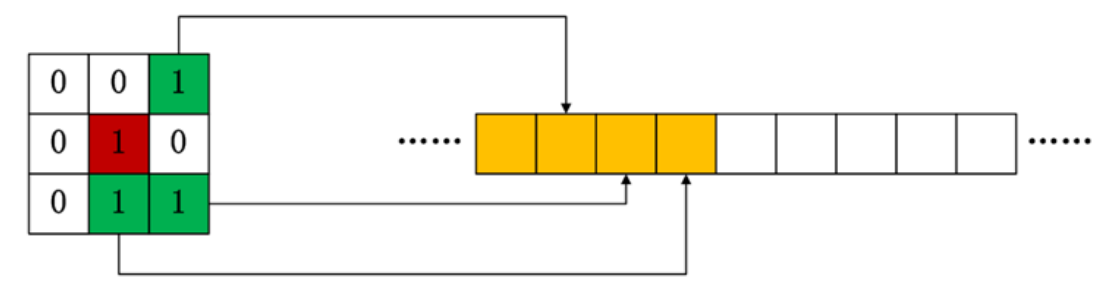

(c)

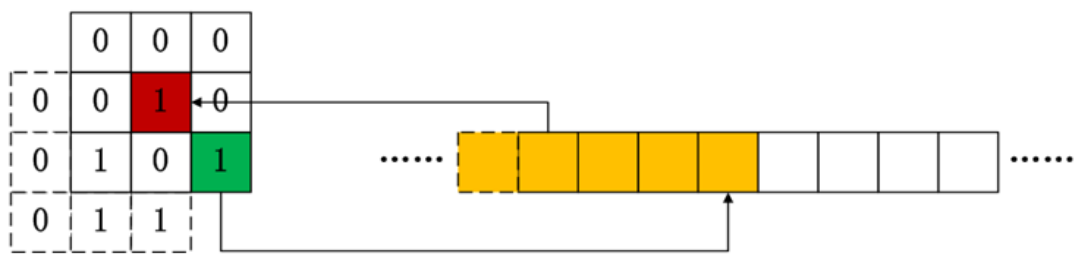

Fig. 2 The algorithm diagram of region growing based on texture feature. (a) select a seed. (b) find the similar cells. (c) take a new cell and continue growing

\subsection{Offshore Ship Detection}

In the sea area segmented by region growing based on texture feature, some candidate regions where the ship targets are likely to exist are captured based on the difference of gray feature between the sea and offshore ships. Otsu algorithm [7], an adaptive threshold segmentation method, is used to extract candidate regions in our algorithm. The algorithm adaptively determines the optimal segmentation threshold of gray image according to the criterion of maximal distance between classes, with good real-time performance and low computational complexity.

After applying the adaptive threshold segmentation many meaningless false alarms still exist in the candidate regions, as gray information is not rich enough to discriminate between offshore ship targets and false alarms in the sea area. There- 
fore we use shape analysis to further eliminate false alarms. Firstly, we fill the small voids and connect the adjacent regions by morphological closing operation, and then the candidate regions that simultaneously meet the constraint conditions (9) will be selected as the final ship targets. Three significant shape features are extracted in this step.

$$
\left\{\begin{array}{c}
t h r_{1} \leq \text { area } \leq t h r_{2} \\
t h r_{3} \leq \text { aspect ratio } \leq t h r_{4} \\
\text { duty ratio }>\text { thr } r_{5}
\end{array}\right.
$$

\subsection{Docked Ship Detection}

Docked ship targets are generally located in the sea-land border region. Their hull superstructure often overlaps the land in the front view so that it gets difficult to distinguish the docked ship targets from complex land background. However, main hull located at sea is easy to identify because of its prominent boundary. We use two bottom boundary fragments as templates to search and match ship targets in the multiscale image instead of the complete hull boundary in order to better tolerate the influence of different types, sizes, perspectives and occlusion. A common reference point (an estimate of the object centroid) is defined according to prior knowledge of ship targets. In order to improve efficiency and reduce computational complexity, we only search the targets in the sea-land border area.

We use Chamfer matching to detect and locate the docked ship targets. Chamfer matching [8] is a popular technique to find the best alignment between two edge images. Fig. 3 shows the two binary boundary templates we established and the multi-templates matching process.

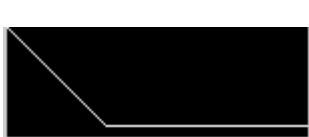

(a)

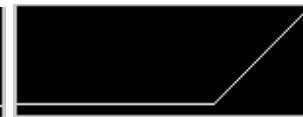

(b)

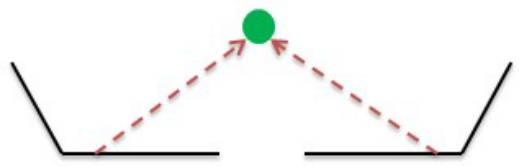

(c)

Fig. 3 The two binary boundary templates: (a) and (b). (c) the multi-templates matching process

\section{Experimental Results}

We tested several texture descriptors for satellite images captured from Google Earth. We choose the best contrast texture feature based on gray histogram which has both strong discrimination and low computational complexity. Fig. 4 indicates contrast (1) has best performance among texture features defined as (1) (8). Its 
convergence boundary is close to the real sea-land boundary, and it costs the least computing time in our experiment.

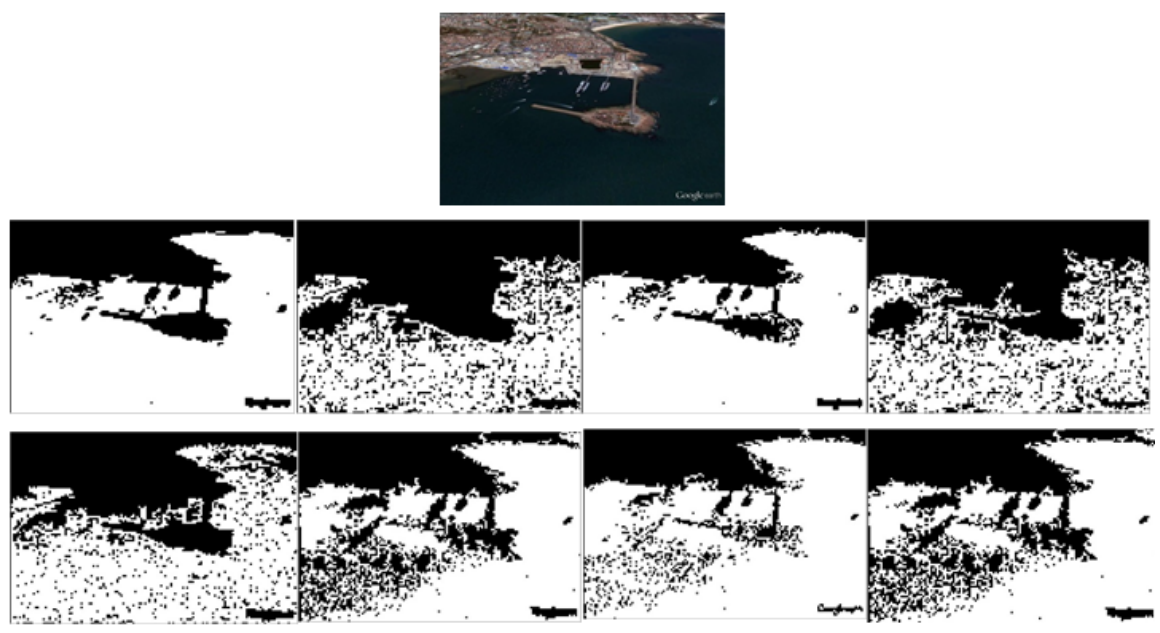

Fig. 4 An example of region growing based on several texture descriptors. The results of region growing based on texture descriptors defined as (1) (4) (2nd row) and (5) (8) (3rd row)

We tested our offshore and docked ship detection algorithm on the real dataset we collected, respectively. For offshore ship detection, we tested 484 images, including 497 offshore targets. There were 528 detections contain 456 true positives and 72 false positives in our experiment. Therefore, the detection rate was $91.75 \%$ and the false positives per image was 0.1488 . The average processing time per image was approximately $180 \mathrm{~ms}$. Our experimental results show that the method combined with adaptive threshold segmentation and shape analysis is able to effectively implement offshore ship detection. The most false alarms are reefs and wharfs stretching to the sea.

For docked ship detection, we tested 165 images, including 165 docked targets. There were 164 detections contain 134 true positives and 30 false positives in our experiment. Therefore, the detection rate was $81.71 \%$ and the false positives per image was 0.1818 . The average processing time per image was approximately 1.5s. Our experimental results show that the salient boundary template matching in the sea-land border area is able to effectively implement docked ship detection. The most false alarms are regions whose shape are similar to the bottom boundary of ship. Some results of ship detection are shown as Fig. 5.

\section{Conclusions}

This paper proposed an algorithm for ship object detection in image with complex harbor background. We tested the performance of several texture features, and region growing based on contrast texture feature was proposed to implement sea- 
land separation. We applied a method combined with adaptive threshold segmentation and shape analysis for offshore ship detection. Furthermore, the salient boundary template matching in the sea-land border area was used for docked ship detection. The experimental results on the real dataset we collected demonstrate the feasibility and effectiveness of our algorithm. In the future work, inter-frame correlation information will be considered and we believe merging the motion information can further reduce false alarms and speed up the process, improving the performance of ship detection in the videos.

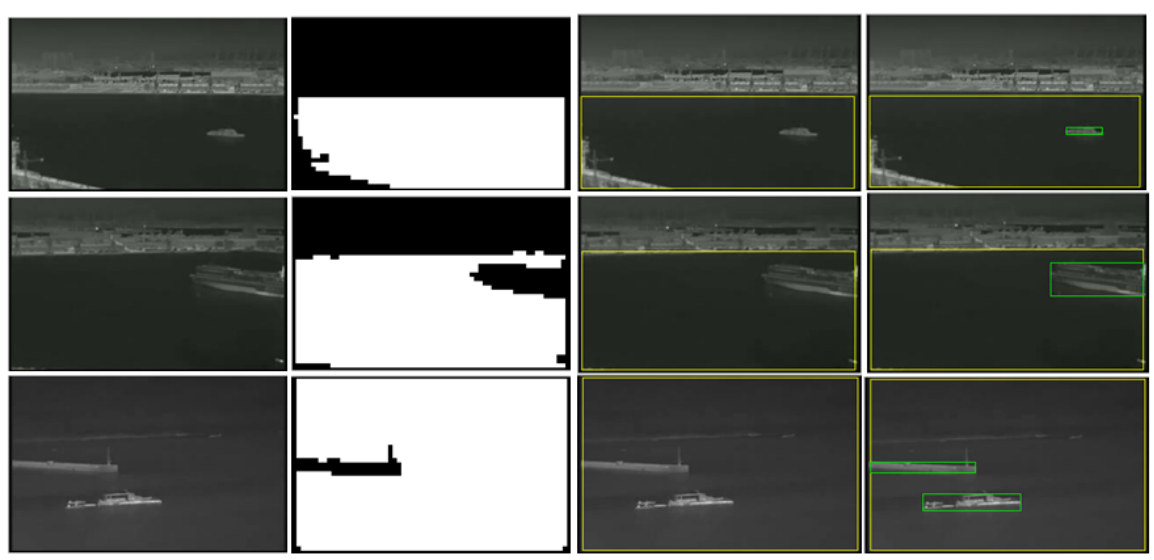

(a) offshore ship detections

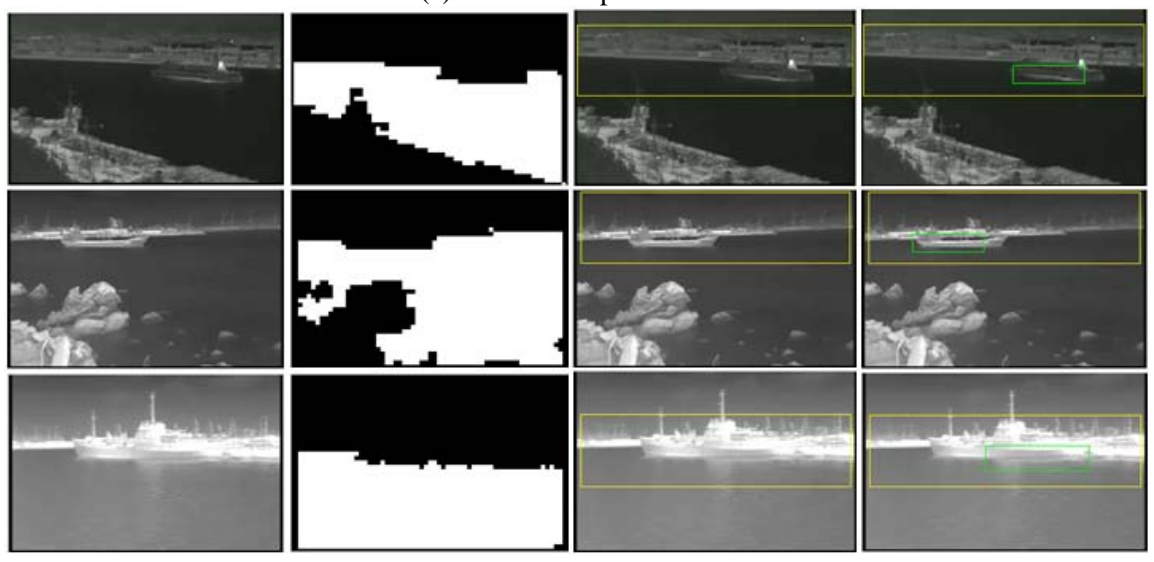

(b) docked ship detections

Fig. 5 Examples of offshore (a) and docked (b) ship detections. 1st column: the harbor images; 2nd column: results of region growing; 3rd column: the extracted sea (a) and sea-land border area (b; 4th column: results of ship detection 


\section{Acknowledgements}

This work was supported by the National High Technology Research and Development Program of China (863 program) under Grant No. 2012AA011004, and the National Natural Science Foundation of China under Grant No. 61071135.

\section{References}

1. Changren Zhu, Hui Zhou, Runsheng Wang, Jun Guo (2010). A novel hierarchical method of ship detection from spaceborne optical image based on shape and texture features. IEEE Transactions on Geoscience and Remote Sensing, 48(9), 3446-3456.

2. Yanqing Wang, Lei Ma, Yuan Tian (2011). State-of-the-art of Ship Detection and Recognition in Optical Remotely Sensed Imagery. Acta Automatica Sinica, 37(9), 1029-1039.

3. Wenyun Huang, Huimin Ma, Shengjin Wang (2009). Recognition algorithm for sea background infrared targets. Journal of Tsinghua University(Science and Technology), 49(10), 1609-1613.

4. Junhua Hu, Shoushi Xu, Hailin Chen, Zhen Zhang (2009). Detection of Ships in Harbor in Remote Sensing Image Based on Local Self-similarity. Journal of Image and Graphics, 14(4), 591-597.

5. Songtao Liu, Huili Wang, Fuliang Yin (2012). Interactive Ship Infrared Image Segmentation Method Based on Graph Cut and Fuzzy Connectedness. Acta Automatica Sinica, 38(7), 17351750.

6. Lining Gao, Fukun Bi, Teng Long, Jian Yang (2011). Ship detection algorithm for optical remote sensing images. Journal of Tsinghua University(Science and Technology), 51(1), 105110.

7. Nobuyuki Otsu (1975). A threshold selection method from gray-level histograms. Automatica, 11(285-296), 23-27.

8. Gunilla Borgefors (1988). Hierarchical chamfer matching: a parametric edge matching algorithm. IEEE Transaction on Pattern Analysis and Machine Intelligence, 10(6), 849-865. 